\title{
Fabricating Functional Materials from Nanomaterial Building Blocks
}

\section{T.W. Chamberlain}

Institute of Process Research and Development, School of Chemistry, University of Leeds, Woodhouse Lane, Leeds LS2 9JT, UK

Corresponding and Presenting Author. E-mail: t.w.chamberlain@leeds.ac.uk

DOI: $10.5185 /$ vpoam.2021.02138

\begin{abstract}
I am focused on the overall vision of controlling, understanding and directing the properties of materials at the atomic level. This enables the intelligent design and fabrication of materials with properties that are tailored to specific applications. My multi-disciplinary approach to real world problems, combines the ability to design and make nanomaterials for targeted application with the advanced spectroscopic tools to fully understand how these materials function. This approach has been exploited to generate functional nanomaterials in a number of areas including; redox flow batteries [1]; multifunctional 1D metal nanowire arrays for gas sensing [2] and nanoparticle catalysts [3].

At the University of Leeds, I have established an industrially-facing research group, applying this unique approach to industrial challenges, working in partnership with a number of companies in a wide variety of areas including; heterogeneous catalyst characterisation and development, continuous nanoparticle synthesis, and automated and high throughput pharmaceutical and agrochemical manufacturing. By harnessing continuous flow platforms and automation it is possible to utilise performance directed optimisation to refine processes with unprecedented levels of efficiency, reducing development time and facilitating scale-up. Specifically, from a nanomaterials perspective, this relates to the fabrication and use of nanoparticle systems but this approach can be exploited in other areas of chemistry, such as pharmaceutical process development.

This talk will outline some of the recent advancements made within the group both in terms of fabrication methodologies and application of nanomaterial systems.
\end{abstract}

\section{References}

1. J. Friedl, M. A. Lebedeva, K. Porfyrakis, U. Stimming, T. W. Chamberlain*, Journal of the American Chemical Society, 2018, 140, 401.

2. P. Clément, X. Xu, C.T. Stoppiello, G.A. Rance, A. Attanzio, J.N. O'Shea, R.H. Temperton, A.N. Khlobystov, K.R.J. Lovelock, J.M. Seymour, R.M. Fogarty, A. Baker, R.A. Bourne, B. Hall, T.W. Chamberlain*, M. Palma*, Angewandte Chemie International Edition, 2019, 58(29), 9928-9932.

3. S. Doherty*, JG Knight, T. Backhouse, RJ Summers, E Abood, W Simpson, W Paget, RA Bourne TW Chamberlain*, R Stones, KRJ Lovelock, J Seymour, M Isaacs, C Hardacre, H Daly, NH Rees, ACS Catalysis, 2019, 9.6, 4777-4791. 


\section{Biography of Presenting Author}

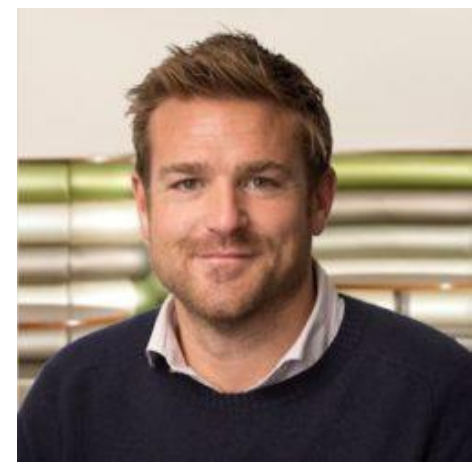

Thomas Chamberlain comes from Matlock, in Derbyshire, and completed an MSci in Chemistry at the University of Nottingham in 2005. He was then awarded a University Interdisciplinary award to study a PhD with Professors Andrei Khlobystov and Neil Champness in Chemistry and Peter Beton in the School of Physics working on the synthesis of novel functional fullerene molecules and the subsequent formation of fullerene/carbon nanotube peapod structures. He received his PhD in 2009 and then joined the Nottingham Nanocarbon group as a post-doctoral research associate studying the use of supramolecular forces, such as van der Waals and H-bonding, to organise molecules in 1D and 2D arrays utilising carbon nanotubes as quasi 1D templates. During this position he established the application of carbon nanotubes as catalytic nanoreactors for the formation of novel molecular and nanostructured products and developed a wide variety of techniques to study the interactions of carbon and metal species at both atomic and bulk length scales.

Dr Chamberlain was appointed as a University Academic Fellow in Nanotechnology for Catalysis at the University of Leeds in 2015. Where he has established his own independent research group within the Institute of Process Research and Development applying his understanding of nanomaterials to both fundamental and applied research challenges. Specifically, there is a focus on the fabrication and application of functional nanomaterials, developing new methods for assembling hybrid nanomaterials, consisting of both molecular and nanoparticle building blocks, and monitoring these processes and the subsequent performance of materials in situ using a variety of spectroscopic techniques. In addition, the group works with flow chemistry and automated optimization systems enabling fundamental understanding in terms of designing and utilizing carbon supported hybrid materials to be harnessed to solve real world problems.

\section{Citation of Video Article}

Vid. Proc. Adv. Mater., Volume 2, Article ID 2102138 (2021)

Full Video Article www.proceedings.iaamonline.org/article/vpoam-2021-02138 